\title{
First-line combination of GELOX followed by radiation therapy for patients with stage IE/IIE ENKTL: An updated analysis with long-term follow-up
}

\author{
LIANG WANG $^{1,2}$, ZHI-HUI WANG $^{3}$, XIAO-QIN CHEN ${ }^{1,2}$, KE-FENG WANG $^{1,2}$, \\ HUI-QIANG HUANG ${ }^{1,4}$ and ZHONG-JUN XIA ${ }^{1,2}$
}

\begin{abstract}
${ }^{1}$ State Key Laboratory of Oncology in South China, Collaborative Innovation Center for Cancer Medicine, Guangzhou, Guangdong; ${ }^{2}$ Department of Hematological Oncology, Sun Yat-sen University Cancer Center, Guangzhou, Guangdong;

${ }^{3}$ Department of Medical Oncology, The Fifth Affiliated Hospital, Sun Yat-sen University, Zhuhai, Guangdong;

${ }^{4}$ Department of Medical Oncology, Sun Yat-sen University Cancer Center, Guangzhou, Guangdong, P.R. China
\end{abstract}

Received September 28, 2014; Accepted May 12, 2015

DOI: $10.3892 / 01.2015 .3327$

\begin{abstract}
In recent years, asparaginase-based chemotherapy regimens have produced excellent short-term efficacy in patients with extranodal natural killer/T-cell lymphoma (ENKTL). However, few long-term outcomes have been reported to date. A phase II clinical trial evaluating the efficacy and safety of a combination of gemcitabine, oxaliplatin and asparaginase (GELOX), followed by radiotherapy (RT) in the treatment of localized ENKTL, was reported by this group in 2012. By the time of the present analysis, detailed information had been collected for all 27 patients in the phase II trial, over an extended follow-up period. The median follow-up time was 63.15 months. The 5-year overall survival and progression-free survival were 85.0 and $74.0 \%$, respectively. Recurrence within the RT field was observed in three patients, and the planning target-volume control rate at 5 years was $88.9 \%$. One patient with confirmed lung invasion who did not respond to autologus stem cell transplantation (ASCT) was successfully treated by salvage therapy with lenalidomide monotherapy, and the EBV DNA load in this individual reflected disease progression and treatment response. No clinically significant late toxicities were identified during follow-up visits. In conclusion, this updated analysis confirmed the long-term benefit of the GELOX regimen followed by RT, and demonstrated a good safety profile for this treatment. This strategy may be one of the most suitable options for the treatment of early stage ENKTL.
\end{abstract}

Correspondence to: Professor Zhong-Jun Xia, Department of Hematological Oncology, Sun Yat-sen University Cancer Center, Building 2, 15th Floor, 651 Dongfeng East Road, Guangzhou, Guangdong 510060, P.R. China

E-mail: xiazhj@21cn.com

Key words: gemcitabine, oxaliplatin, asparaginase, extranodal natural killer/T-cell lymphoma, survival

\section{Introduction}

Extranodal natural killer/T-cell lymphoma (ENKTL), an Epstein-Barr virus (EBV)-associated lymphoma, has a racial and geographical predisposition, and accounts for $5-10 \%$ of all malignant lymphomas in China (1). For patients with stage IE ENKTL without any risk factors, radiotherapy (RT) alone has been shown to achieve excellent outcomes (2). Such risk factors include an age of $>60$ years, B symptoms, an Eastern Cooperative Oncology Group performance status of $\geq 2$, elevated lactate dehydrogenase levels, regional node involvement, local tumor invasion (bone or skin), high Ki-67 staining on histology, and an EBV DNA titer of $\geq 6.1 \times 10^{7}$ copies $/ \mathrm{ml}$ (3). However, for individuals with stage IE disease with risk factors or those with stage IIE ENKTL, an increasing number of studies have recommended concurrent (4) or sequential (5) chemoradiation, in order to reduce the relapse rate and improve the long-term outcome.

Overexpression of multidrug-resistant (MDR) genes, such as MDR-1, MRP, LRP and BCRP, leads to increased levels of P-glycoprotein in NK/T-cell lymphoma cells. P-glycoprotein actively exports doxorubicin and vincristine, which are the main components of the CHOP chemotherapy regimen, subsequently leading to reduced treatment efficacy and worse survival outcomes $(6,7)$. Thus, due to a high expression of MDR genes, ENKTL is resistant to anthracycline-based chemotherapy (8). L-asparaginase has been found to exhibit a specific anticancer mechanism; it can hydrolyze and exhaust serum asparagines in NK/T-cell lymphoma cells, which are unable to synthesize L-asparagines, subsequently producing an anticancer effect (9). Notably, asparaginase is not affected by P-glycoprotein $(10,11)$, as it is not a substrate of P-glycoprotein and thus is not exported from tumor cells (12). A number of studies have incorporated asparaginase into existing chemotherapy regimens and have achieved promising short-term results $(5,9,13)$. However, to the best of our knowledge, no long-term efficacy and safety data of asparaginase-based treatment have been reported, due to relatively short follow-up periods. As previously reported (5), this 

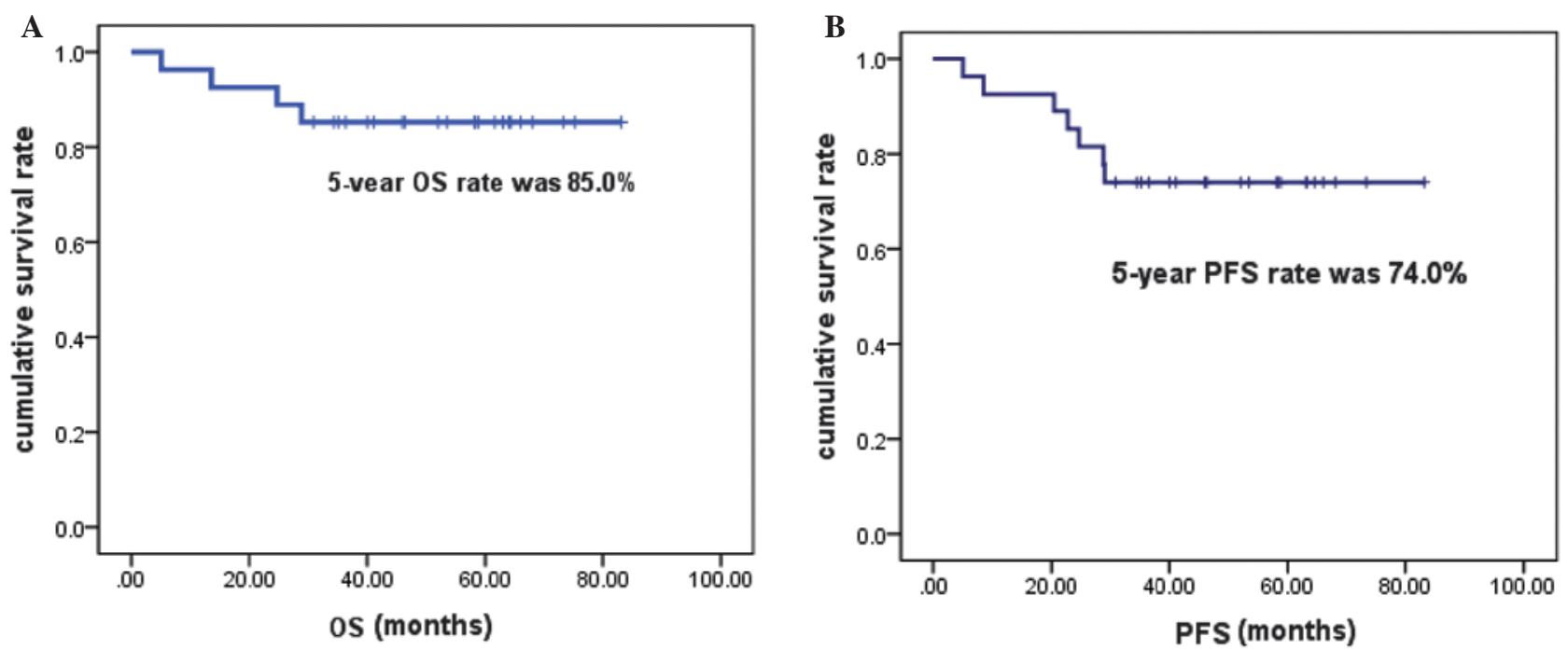

Figure 1. (A) OS and (B) PFS of 27 patients treated with the gemcitabine, oxaliplatin and asparaginase regimen followed by radiotherapy. OS, overall survival; PFS, progression-free survival.

group previously conducted a phase II clinical trial, evaluating the efficacy and safety of a combination of gemcitabine, oxaliplatin and asparaginase (GELOX), followed by RT in the treatment of localized ENKTL, for which follow-up has been ongoing since 2008. At the end of treatment, the overall response rate (ORR) was $96.3 \%$, with a complete remission (CR) rate of $74.1 \%$, and side effects were well tolerated. In the initial analysis, the median follow-up time was 27 months, and 2-year overall survival (OS) and progression-free survival (PFS) were each $86.0 \%$. As of 6th July 2014, the majority of patients had been followed up for $>5$ years. The current study therefore reports long-term outcomes of GELOX-based treatment. Furthermore, patterns of failure, salvage treatments and the involvement of EBV DNA in the early detection of relapse are discussed.

\section{Patients and methods}

Patients. From January 2008 to July 2011, 27 patients, who were newly diagnosed with stage IE or stage IIE ENKTL, were enrolled. The primary site for all patients was the upper aerodigestive tract, and the patients were enrolled regardless of risk factors. Written informed consent was obtained from all patients prior to enrollment, and the study was approved by the Sun Yat-sen University Cancer Center Research Ethics Board. Inclusion and exclusion criteria were as previously reported (5).

Treatment. Patients were treated with an initial GELOX regimen. Following $\geq 2$ cycles of GELOX, patients were referred for RT. Subsequently, they received 2-4 cycles of GELOX within 1 week of the completion of RT, resulting in a maximum total of 6 cycles of GELOX. The dosage of the GELOX regimen and RT were as previously reported (5).

Statistical analysis. PFS was calculated from the date of diagnosis to the date of identification of disease progression, and was censored at the date of the last follow-up visit. OS was calculated from the date of diagnosis to the date of death from any cause, and was censored at the date of the last follow-up visit. All statistical analysis was performed using PASW Statistics 18.0 software (Apache Software Foundation, Forest Hill, MD, USA). Survival outcomes were assessed the Kaplan-Meier method and log-rank test. Differences between the results of comparative tests were considered significant if the two-sided P-value was $<0.05$.

\section{Results}

Patient characteristics and initial findings. 27 patients were enrolled in the phase II clinical trial (mean age, 47 years; range, 21-74 years). Of these, 66.7\% (18/27) had stage IE disease, and all patients had an International Prognostic Index score of 0-1. As previously reported, the ORR was $96.3 \%$ at the completion of first-line treatment, with a CR of $74.1 \%$ and a PR of $22.2 \%$.

Updated survival data. The data used for this analysis were updated in July 2014, and detailed follow-up information was available for all 27 patients. The median follow-up time was 63.15 months (range, 30.92-83.15 months). The 5-year OS rate was $85.0 \%$, and the 5-year PFS rate was $74.0 \%$ (Fig. 1). Four patients were not alive at the end of the follow-up period, and no further mortality occurred following the initial analysis. A total of 7 patients exhibited disease progression, of whom 4 did not survive, due to failure of salvage therapy. Recurrence within the RT field was observed in 3 patients, and thus the planning target-volume control rate at 5 years was $88.9 \%$ (24 out of 27 patients). Two patients with locoregional relapse responded to subsequent GELOX and pegaspargase-based chemotherapy and achieved a CR. One patient developed a lung recurrence following locoregional relapse. This was a male patient, aged 41, who was diagnosed with stage IE ENKTL in April 2009. He was treated with 6 cycles of GELOX followed by RT (56 Gy) and subsequently achieved CR. However, he developed locoregional relapse in September 2010. Seven cycles of a combination of pegaspargase and IMVP-16 (ifosfamide, methotraxate and etoposide) were administered and unconfirmed CR was attained in the locoregional relapse site. 
A

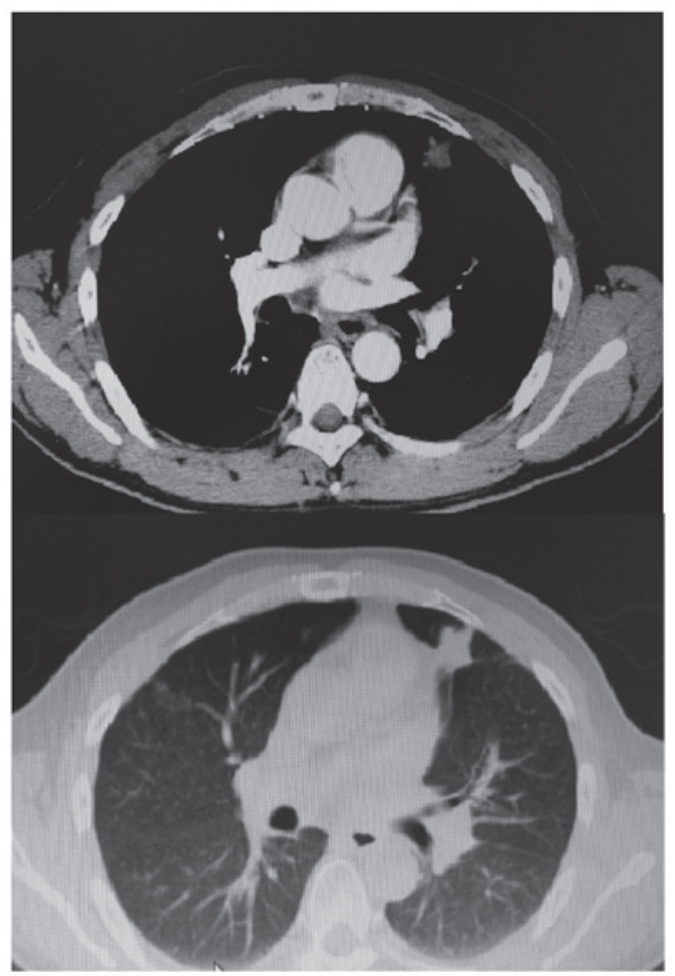

B

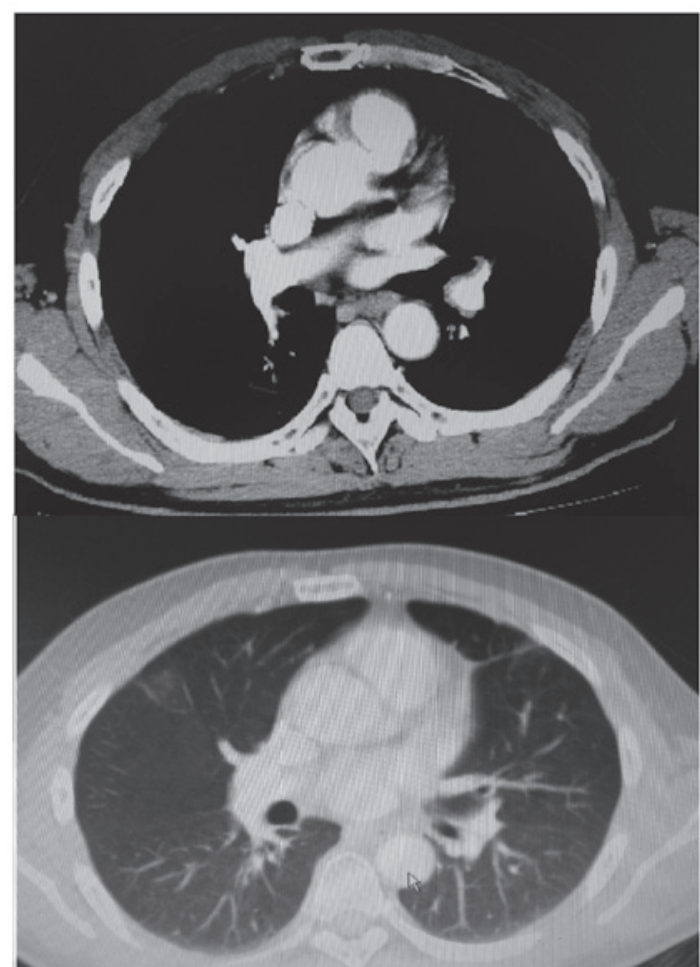

Figure 2. (A) CT scan performed at 6 months post-ASCT, showing a 25x $21 \mathrm{~mm}$ solid nodule in the left lung, which indicated disease progression. (B) CT scan performed at 4 months after lenalidomide treatment, showing complete remission of the left lung lesion. Upper images, mediastinal window; lower images, lung window.

However, a single small solid nodule, $8 \times 7 \mathrm{~mm}$ in size, was identified in the left lung during the salvage chemotherapy, and a biopsy confirmed the diagnosis of ENKTL. Due to the relapsed/refractory nature of this patient's disease, autologous stem cell transplantation (ASCT) was performed on 7th May 2011 (day 0), using BEAM as the conditioning regimen (carmustine $300 \mathrm{mg} / \mathrm{m}^{2}$, day -6 ; cytarabine $200 \mathrm{mg} / \mathrm{m}^{2}$, twice daily, days -5 to -2 ; VP-16 $200 \mathrm{mg} / \mathrm{m}^{2}$, days -5 to -2 ; and melphalan $140 \mathrm{mg} / \mathrm{m}^{2}$, day -1). As shown in Fig. 2A, at 6 months post-ASCT, a $25 \times 21 \mathrm{~mm}$ solid nodule was observed in the left lung, which was significantly larger than the nodule had been pre-ASCT. At this time, the EBV DNA load was $4.01 \times 10^{3}$ copies $/ \mathrm{mL}$, which indicated persistence of ENKTL. Lenalidomide was then administered as salvage therapy, at a dosage of $15 \mathrm{mg} / \mathrm{d}$, from days 1 to 21 , followed by a rest for 1 week. Treatment was well tolerated. Four months after lenalidomide treatment, the CT scan demonstrated no discernible lesion in the left lung (Fig. 2B) and the EBV DNA load was undetectable. Lenalidomide was administered as maintenance therapy for a total of 2 years, and regular follow-up CT scans demonstrated no evidence of relapse. The patient was alive without disease at the last follow-up visit.

Toxicity. No clinically significant late-onset toxicities were observed during follow-up visits. As reported previously, <grade 3 mucositis and dermatitis occurred frequently during $\mathrm{RT}$, and resolved in all patients without compromising quality of life [assessed using the European Organization for Research and Treatment of Cancer Quality of Life Questionnaire, QLQ-C30 (14)]. No treatment-related mortality occurred.
However, a 52 year-old old female patient developed left breast intraductal papilloma with atypical hyperplasia 4 years after the completion of treatment for ENKTL. No causality was found and this finding may be unrelated to the treatment she received.

\section{Discussion}

It has been reported that ENKTL is resistant to anthracycline-base chemotherapy regimens due to high expression of P-glycoprotein (8). L-asparaginase-based regimens, such as combined dexamethasone, methotrexate, ifosfamide, L-asparaginase and etoposide (SMILE) (9), and combined asparaginase, methotrexate and dexamethasone (AspaMetDex) (13), have produced promising survival outcomes. Since January 2008, this group has been conducting a phase II clinical trial, evaluating the efficacy of GELOX in the treatment of early stage ENKTL (5). As previously reported, the short-term outcomes were significantly better than those of CHOP-based regimens (15). In this updated analysis, with a median follow-up time of 63.15 months, the 5-year PFS and OS were 74.0 and $85.0 \%$, respectively, which were significantly better than those of previous CHOP-based results, in which 5-year OS rate was $48.0 \%$ (15). The Japan Clinical Oncology Group Study (JCOG0211) (16) updated the long-term outcomes of concomitant chemoradiotherapy using the DeVIC regimen (dexamethasone, etoposide, ifosfamide and carboplatin) for localized ENKTL, and the 5-year PFS and OS were $63 \%$ and $70 \%$, respectively, which appear to be inferior to the results of the present study. In addition, in comparison with the safety profiles of the current study (5), a greater number of grade 3/4 hematological events and RT-related adverse events 
were documented in the JCOG0211 study (4). Furthermore, no overt late toxicities were observed in the present group, while in the JCOG0211 group, one patient developed a grade 4 late RT adverse event (perforation of the nasal skin, which required plastic surgery) and 11 patients developed grade 1 or 2 late RT adverse effects of the eye (16). Therefore, GELOX-based sequential chemoradiotherapy appears to be highly efficacious and safe in the treatment of localized ENKTL. Currently, this groups is undertaking a phase II clinical trial, evaluating the efficacy and safety of concomitant chemoradiotherapy using the GELOX regimen, which aims to investigate whether this strategy may further reduce the relapse rate, while maintaining acceptable toxicity profiles (NCT02080234; http://clinicaltrials.gov).

In the present cohort, three patients who had relapsed, responded to subsequent asparaginase-based chemotherapy, indicating that asparaginase may be used as salvage treatment, regardless of prior exposure. Recently, a number of studies have demonstrated that the tumor microenviroment may be important in tumorigenesis and tumor progression (17). Lin et al (18) showed that high expression of tumor-associated macrophages predicted a poor prognosis in patients with ENKTL, indicating that therapies targeting the tumor microenviroment may be effective in ENKTL. As shown in Fig. 2, the lung lesion in a patient with relapsed disease, was refractory to ASCT, while it responded well to lenalidomide monotherapy, indicating the potential efficacy of immunomodulatory drugs in the treatment of ENKTL. This group is also currently undertaking a phase III trial, comparing the efficacy of a pegaspargase-Gemox regimen followed by thalidomide, with that of the AspaMetDex regimen in patients with de novo or relapsed ENKTL, with the aim of investigating the role of thalidomide as maintenance therapy in ENKTL (NCT02085655; http://clinicaltrials.gov).

A number of studies have indicated that the plasma EBV DNA load at presentation may predict survival outcomes $(19,20)$. Unfortunately, measurement of the EBV DNA load was initially not routinely conducted. Therefore, an analysis of EBV DNA load was not included in the first analysis of the current clinical trial. Over the past 2 years, this strategy was modified to test the EBV DNA load in all patients with ENKTL at each follow-up visit. In the patient with relapsed disease, who was treated with ASCT and lenalidomide, the EBV DNA load was 0 copy/ml on 28th September 2011 (4 months post-ASCT). It increased to $4.01 \times 10^{3}$ copy $/ \mathrm{ml}$ on 26th December 2011 (6 months post-ASCT), at which time the $\mathrm{CT}$ scan revealed progression of the existing lung lesion. The EBV DNA load had returned to 0 copy/ml on 17 th December 2012 (10 months after lenalidomide treatment), and the CT scan at that time demonstrated no detectable lesions in the lung, thereby confirming CR. Thus, the EBV DNA load may be used as an surrogate biomarker for early detection of relapsed disease, in addition to a reflection of treatment response. The two ongoing clinical trials, have implemented required EBV DNA load testing, and may validate the role of EBV DNA load measurement in monitoring patient with ENKTL.

In conclusion, the present updated analysis confirmed the long-term benefit of GELOX regimen followed by RT, and demonstrated good safety profiles with this approach. This strategy may currently be one of the most suitable options for treatment of early stage ENKTL.

\section{Acknowledgements}

The authors would like to thank the doctors of Sun-Yat Sen University Cancer Center, for recruiting the patients involved in this study, and the pathologists of Sun-Yat Sen University Cancer Center for their assistance. This study received financial support from the National Natural Science Foundation of China (grant no. 81400159), the Young Teachers' Cultivation Project of Sun Yat-sen University (grant no. 12ykpy54) and the Outstanding Young Talents Project of Sun Yat-sen University Cancer Center (grant no. 04190101).

\section{References}

1. Li YX, Liu QF, Fang H, Qi SN, Wang H, et al: Variable clinical presentations of nasal and Waldeyer ring natural killer/T-cell lymphoma. Clin Cancer Res 15: 2905-2912, 2009.

2. Li YX, Wang H, Jin J, Wang WH, Liu QF, et al: Radiotherapy alone with curative intent in patients with stage I extranodal nasal-type NK/T-cell lymphoma. Int J Radiat Oncol Biol Phys 82: 1809-1815, 2012.

3. Kohrt H, Lee M and Advani R: Risk stratification in extranodal natural killer/T-cell lymphoma. Expert Rev Anticancer Ther 10: 1395-1405, 2010 .

4. Yamaguchi M, Tobinai K, Oguchi M, Ishizuka N, Kobayashi Y, Isobe Y, et al: Phase I/II study of concurrent chemoradiotherapy for localized nasal natural killer/T-cell lymphoma: Japan Clinical Oncology Group study JCOG0211. J Clin Oncol 27: 5594-5600, 2009.

5. Wang L, Wang ZH, Chen XQ, Li YJ, Wang KF, et al: First-line combination of gemcitabine, oxaliplatin, and L-asparaginase (GELOX) followed by involved-field radiation therapy for patients with stage IE/IIE extranodal natural killer/T-cell lymphoma. Cancer 119: 348-355, 2013.

6. Baekelandt M, Lehne G, Tropé CG, Szántó I, Pfeiffer P, Gustavssson B and Kristensen GB: Phase I/II trial of the multidrug-resistance modulator valspodar combined with cisplatin and doxorubicin in refractory ovarian cancer. J Clin Oncol 19: 2983-2993, 2001.

7. Shiraki N, Hamada A, Ohmura T, Tokunaga J, Oyama N and Nakano M: Increase in doxorubicin cytotoxicity by inhibition of P-glycoprotein activity with lomerizine. Biol Pharm Bull 24: 555-557, 2001.

8. Wang B, Li XQ, Ma X, Hong X, Lu H and Guo Y: Immunohistochemical expression and clinical significance of P-glycoprotein in previously untreated extranodal NK/T-cell lymphoma, nasal type. Am J Hematol 83: 795-799, 2008.

9. Yamaguchi M, Kwong YL, Kim WS, Maeda Y, Hashimoto C, Suh C, Izutsu K, Ishida F, Isobe Y, Sueoka E, et al: Phase II study of SMILE chemotherapy for newly diagnosed stage IV, relapsed, or refractory extranodal natural killer (NK)/T-cell lymphoma, nasal type: The NK-Cell Tumor Study Group study. J Clin Oncol 29: 4410-4416, 2011.

10. Martin JK, Sun W, Moraga-A D, Schuster SM and Wylie DE: An investigation into the mechanism of L-asparaginase resistance in L5178Y murine leukemia cells. Amino Acids 5: 51-69, 1993.

11. Tse E and Kwong YL: How I treat NK/T-cell lymphomas. Blood 121: 4997-5005, 2013.

12. van den Berg H: Asparaginase revisited. Leuk Lymphoma 52: 168-178, 2011.

13. Jaccard A, Gachard N, Marin B, Rogez S, Audrain M, et al: Efficacy of L-asparaginase with methotrexate and dexamethasone (AspaMetDex regimen) in patients with refractory or relapsing extranodal NK/T-cell lymphoma, a phase 2 study. Blood 117: 1834-1839, 2011.

14. Chie WC, Yang CH, Hsu C and Yang PC: Quality of life of lung cancer patients: Validation of the Taiwan Chinese version of the EORTC QLQ-C30 and QLQ-LC13. Qual Life Res 13: 257-262, 2004.

15. Wang L, Xia ZJ, Huang HQ, et al: Cyclophosphamide, doxorubicin, vincristine, and prednisone (CHOP) in the treatment of stage IE/IIE extranodal natural killer/T cell lymphoma, nasal type: 13-year follow-up in 135 patients. Int J Hematol 96: 617-623, 2012. 
16. Yamaguchi M, Tobinai K, Oguchi M, Ishizuka N, Kobayashi Y, Isobe $\mathrm{Y}$ et al: Concurrent chemoradiotherapy for localized nasal natural killer/T-cell lymphoma: An updated analysis of the Japan clinical oncology group study JCOG0211. J Clin Oncol 30: 4044-4046, 2012.

17. Rosenquist R, Davi F and Ghia P: The microenvironment in lymphomas - dissecting the complex crosstalk between tumor cells and 'by-stander' cells. Semin Cancer Biol 24: 1-2, 2014.

18. Lin ZX, Bai B, Cai QC, et al: High numbers of tumor-associated macrophages correlate with poor prognosis in patients with mature T- and natural killer cell lymphomas. Med Oncol 29: 3522-3528, 2012.
19. Kim HS, Kim KH, Kim KH, Chang MH, Ji SH, et al: Whole blood Epstein-Barr virus DNA load as a diagnostic and prognostic surrogate: Extranodal natural killer/T-cell lymphoma. Leuk Lymphoma 50: 757-763, 2009.

20. Suzuki R, Yamaguchi M, Izutsu K, Yamamoto G, Takada K, Harabuchi Y, et al: Prospective measurement of Epstein-Barr virus-DNA in plasma and peripheral blood mononuclear cells of extranodal NK/T-cell lymphoma, nasal type. Blood 118: 6018-6022, 2011. 